\title{
A Deadly Complication of Systemic Lupus Erythematosus
}

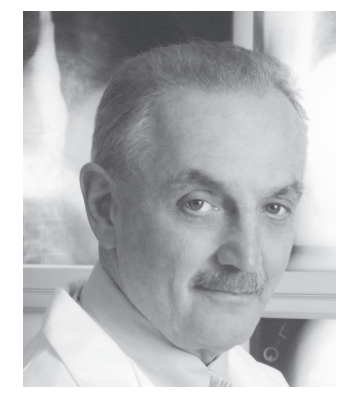

Diffuse alveolar hemorrhage (DAH), often a catastrophic event resulting in acute respiratory failure, occurs in the setting of the collagen vascular diseases, in particular systemic lupus erythematosus (SLE). There are a number of other systemic conditions that may result in this complication, including any of the systemic vasculitides, antibasement membrane antibody disease, medications that result in vasculitis, antiphospholipid syndrome, various coagulopathies, thrombocytopenia from any cause, and less frequently, severe viral infections ${ }^{1}$.

In this issue of The Journal Martinez-Martinez, et al describe 50 subjects with SLE, both adults and juveniles, who experienced 57 episodes of $\mathrm{DAH}^{2}$. This represents the largest collection of patients with this SLE complication. There were no differences in outcomes between juveniles ( $<$ $18 \mathrm{yrs}$ ) and adults. In the adult group $90 \%$ were women and the mean age for the entire cohort was 23 years. Overall mortality was $42 \%$, and not unexpectedly, the sicker patients, i.e., those requiring mechanical ventilation and those with infections, renal dysfunction, hypocomplementemia, and thrombocytopenia, had the worst outcomes. Because this was a retrospective analysis, there was no standardization of treatment before or during the DAH episode. The results reported in this study correspond to prior studies, i.e., survival depending on disease severity and the presence of infection ${ }^{3,4,5}$. It is unclear, however, whether the infections resulted from the SLE treatment prior to the DAH episode or occurred following treatment for this complication. It is unlikely, however, that diffuse pneumonia alone accounts for DAH in SLE. It is known, however, that in several cases of influenza and severe acute respiratory syndrome not associated with SLE, DAH can occur $^{6}$.

There are several lung histology findings underlying diagnosis of DAH in SLE. The first and likely most frequent is pulmonary capillaritis. This represents a small vessel vasculitis of the lung involving capillaries, arterioles, and venules. This histologic picture is similar to what occurs in
DAH that complicates antineutrophil cytoplasmic antibody positive and negative systemic vasculitides ${ }^{7}$. The second is organizing diffuse alveolar damage. This is the lung injury pattern that underlies the acute respiratory distress syndrome, idiopathic acute interstitial pneumonia (the Hamman Rich syndrome) and likely what was previously referred to as acute lupus pneumonitis ${ }^{8}$. The last is bland pulmonary hemorrhage, in which there are no inflammatory changes in the lung but rather blood and hemosiderin-laden macrophages filling the alveolar spaces. This same picture occurs in idiopathic pulmonary hermosiderosis and with DAH of the coagulopathies ${ }^{1}$. In all 3 histologic pictures there is injury to the shared basement membrane between alveolar capillaries and epithelium, with leakage of blood into the alveolar spaces. It is estimated that $2 \%$ of patients with SLE will develop DAH, and this complication accounts for up to $4 \%$ of all pulmonary hospital admissions in $\mathrm{SLE}^{3}$. Most admissions for SLE pulmonary-associated complications are due to bacterial and viral pneumonias and thromboembolic disease.

The article by Martinez-Martinez, et al confirms the high mortality of DAH in SLE, which is most often due to respiratory failure. It also confirms the association of DAH with lupus nephritis. As expected, the most common demographic affected are younger women. They also noted that only $57 \%$ of these patients reported hemoptysis. This is not unusual, as up to $33 \%$ of subjects with DAH from all causes fail to report this symptom ${ }^{7}$. Pulmonologists always consider the possibility of DAH from any etiology in any patient who presents with more or less diffuse pulmonary infiltrates and a falling hemoglobin level. In such patients, bronchoalveolar lavage (BAL) is performed. If $\mathrm{BAL}$ demonstrates increasing red blood cell counts in sequential lavages, the diagnosis of DAH is established but not the etiology or the underlying pathology. BAL was not routinely performed, and in some cases premorbid serum hemoglobin levels were unknown in the Martinez-Martinez series.

See Diffuse alveolar hemorrhage and SLE, page 1656

Personal non-commercial use only. The Journal of Rheumatology Copyright (C) 2014. All rights reserved. 
This brings us to an important point: In the Martinez-Martinez, et al article, 35\% of the episodes of DAH represented the initial manifestation of SLE. This supports all prior series of this complication in $\mathrm{SLE}^{3,4,5}$. Another important issue that should be noted: Once a DAH episode occurs in SLE, reoccurrences are sometimes a continuing problem. Unfortunately, neither the literature nor the current study reports any tested treatment protocol. Because pulmonary capillaritis underlies DAH, similarly to what occurs in the systemic vasculitides, high-dose intravenous glucocorticoids, cyclophosphamide, rituximab, and plasmapheresis are the usual treatments initiated $9,10,11$. Other case reports describe the effective use of intravenous immunoglobulin. In uncontrolled DAH with severe respiratory failure, extracorporeal membrane oxygenation and activated factor VIII to control hemorrhage have been successful in several reports ${ }^{12,13}$.

Fortunately, DAH in SLE, although often fatal, represents an uncommon complication. BAL establishes the DAH diagnosis, and the lavagate can also be evaluated for infectious agents. Early recognition and prompt treatment likely offer the best chance for improved survival.

\section{MARVIN I. SCHWARZ, MD} University of Colorado Denver, Pulmonary Sciences and Critical Care Medicine, Aurora, Colorado, USA.

Address correspondence to Dr. Schwarz, 12700 East 19th Ave. C272, Aurora, Colorado 80045, USA. E-mail: Marvin.Schwarz@UCDenver.edu

\section{REFERENCES}

1. Lara A, Schwarz MI. Diffuse alveolar hemorrhage. Chest 2010;137:1164-71

2. Martinez-Martinez MU, Sturbaum AK, Alcocer-Varela J, Merayo-Chalico J, Gómez-Martin D, Gómez-Bañuelos JdJE, et al. Factors associated with mortality and infections in patients with systemic lupus erythematosus with diffuse alveolar hemorrhage. J Rheumatol 2014;41:1656-61.
3. Zamora MR, Warner ML, Tudor R, Schwarz MI. Diffuse alveolar hemorrhage and systemic lupus erythematosus (SLE): clinical presentation, histology, survival and outcome. Medicine 1997;76:192-202.

4. Raj R, Murin S, Matthay RA, Wiedemann HP. Systemic lupus erythematosus in the intensive care unit. Crit Care Clin 2002;18:781-803.

5. Santos-Ocampo AS, Mandell BF, Fessler BJ. Alveolar hemorrhage in systemic lupus erythematosus. Chest 2000;118:1083-90.

6. Marchiori E, Zanetti G, Hochhegger B. Diffuse alveolar hemorrhage in infectious diseases. Chest 2011;139:228.

7. Schwarz MI, Brown KK. Small vessel vasculitis of the lungs. Thorax 2000;55:502-10.

8. Matthay RA, Schwarz MI, Petty TL, Stanford RE, Gupta RC, Sahn SA, et al. Pulmonary manifestations of systemic lupus erythematosus: review of twelve cases of acute lupus pneumonitis. Medicine 1975;54:397-409.

9. Narshi CB, Haider S, Ford CM, Isenberg DA, Giles IP. Rituximab as early therapy for pulmonary haemorrhage in systemic lupus erythematosus. Rheumatology 2010;49:392-4.

10. Nellessen CM, Poge U, Brensing KA, Sauerbruch T, Klehr H, Rabe C. Diffuse alveolar haemorrhage in a systemic lupus erythematosus patient successfully treated with rituximab: a case report. Nephrol Dial Transplant 2008;23:385-6.

11. Claridge S, Das P, Dorling A, Robson M. Plasmapheresis as rescue therapy for systemic lupus erythematosus-associated diffuse alveolar haemorrhage. BMJ Case Reports 2011; doi:10.1136/bcr.02.2011.3893 [serial on the Internet. Accessed June 5, 2014.] Available from: http://casereports.bmj.com/content/2011/bcr.02.2011.3893.abstract

12. Claudio CP, Charbonney E, Durand M, Kolan C, Laskine M. Extracorporeal membrane oxygenation in diffuse alveolar hemorrhage secondary to systemic lupus erythematosus. J Clin Med Res 2014;2:145-8.

13. Patel JJ, Lipchik R. Systemic lupus - induced diffuse alveolar hemorrhage treated with extracorporeal membrane oxygenation. J Intensive Care Med 2012;29:104-9.

J Rheumatol 2014;41:1571-2; doi:10.3899/jrheum.140613 